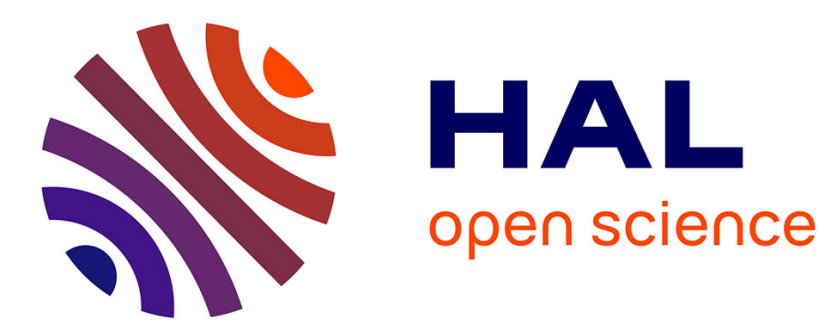

\title{
Psi_B-energy operator and cross-power spectral density
} Abdel-Ouahab Boudraa, Thierry Chonavel, Jean-Christophe Cexus

\section{To cite this version:}

Abdel-Ouahab Boudraa, Thierry Chonavel, Jean-Christophe Cexus. Psi_B-energy operator and crosspower spectral density. Signal Processing, 2014, 94, pp.236-240. 10.1016/j.sigpro.2013.05.022 . hal01088196

\section{HAL Id: hal-01088196 https://hal.science/hal-01088196}

Submitted on 27 Nov 2014

HAL is a multi-disciplinary open access archive for the deposit and dissemination of scientific research documents, whether they are published or not. The documents may come from teaching and research institutions in France or abroad, or from public or private research centers.
L'archive ouverte pluridisciplinaire $\mathbf{H A L}$, est destinée au dépôt et à la diffusion de documents scientifiques de niveau recherche, publiés ou non, émanant des établissements d'enseignement et de recherche français ou étrangers, des laboratoires publics ou privés. 


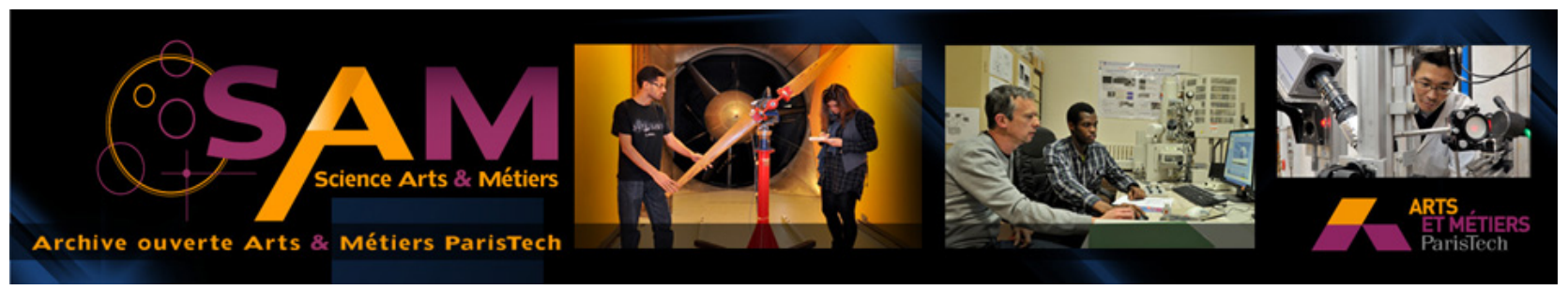

Science Arts \& Métiers (SAM)

is an open access repository that collects the work of Arts et Métiers ParisTech researchers and makes it freely available over the web where possible.

This is an author-deposited version published in: http://sam.ensam.eu Handle ID: .http://hdl.handle.net/10985/8999

\section{To cite this version :}

Abdel-Ouahab BOUDRAA, Thierry CHONAVEL, Jean-Christophe CEXUS - Psi B-energy operator and cross-power spectral density - Signal Processing - Vol. 94, p.236- $\overline{2} 40$ - 2014 


\title{
$\Psi_{\mathrm{B}}$-energy operator and cross-power spectral density
}

\author{
Abdel-Ouahab Boudraa ${ }^{\mathrm{a}, *}$, Thierry Chonavel $^{\mathrm{b}}$, Jean-Christophe Cexus ${ }^{\mathrm{c}}$ \\ a IRENav, Ecole Navale, BCRM Brest, CC 600, 29240 Brest Cedex 9, France \\ b Lab-Sticc, Télécom Bretagne, Technopole Brest-Iroise, 29238 Brest Cedex 3, France \\ ${ }^{\mathrm{c}}$ Lab-Sticc, ENSTA-Bretagne, 2 rue François Verny, 29806 Brest, France
}

Keywords:

$\Psi_{\mathrm{H}}$-energy operator

Cross- $\Psi_{\mathrm{B}}$-energy operator

Teager-Kaiser energy operator

Cross-power spectral density

\begin{abstract}
A B S T R A C T
In this paper we consider the hermitian extension of the cross- $\Psi_{\mathrm{B}}$-energy operator that we will denote by $\Psi_{\mathrm{H}}$. In addition, cross energy terms are formalized through multivariate signals representation. We investigate the connection between the interaction energy function of $\Psi_{\mathrm{H}}$ and the cross-power spectral density (CPSD) of two complex valued signals. In particular, this link permits to use this operator for estimating the CPSD. We illustrate the interest of $\Psi_{\mathrm{H}}$ as a similarity between a pair of signals in frequency domain on synthetic and real data.
\end{abstract}

\section{Introduction}

Since its introduction, the cross- $\Psi_{\mathrm{B}}$-energy operator [1] has been used in different domains, including time series analysis [2], gene time series expression data clustering [3], wave equation [4], transient detection [5], time delay estimation [6] or time-frequency analysis [7]. These applications show that $\Psi_{\mathrm{B}}$, which is a complex and symmetric version of the cross-Teager-Kaiser energy operator [8], is well suited for processing of non-stationary signals.

In this paper, we introduce an hermitian version of $\Psi_{\mathrm{B}}$ that we denote by $\Psi_{\mathrm{H}}$. In particular, it contains all the information in $\Psi_{\mathrm{B}}$ and has a more compact expression. In addition, its hermitian structure makes it quite natural for handling complex signals. Then, we establish the connection between $\Psi_{\mathrm{H}}$ and the cross-power spectral density (CPSD). This function is a fundamental and powerful tool to investigate an unknown second order relationship between two signals (or time series) in the frequency domain [9]. This connection involves an interesting

\footnotetext{
* Corresponding author. Tel.: +332 982340 37; fax: +332 98233857 .

E-mail address: boudra@ecole-navale.fr (A.-O. Boudraa).
}

relationship and a simple way to estimate the CPSD and its second order power moment. Note that all the results presented here for $\Psi_{\mathrm{H}}$ also hold for $\Psi_{\mathrm{B}}$.

\section{Cross spectral density and $\Psi_{\mathrm{H}}$ operator}

For two complex-valued signals $x_{t}$ and $y_{t}, \Psi_{\mathrm{B}}$ operator is defined as follows [1]:

$$
\begin{aligned}
& \Psi_{\mathrm{B}}\left[x_{t}, y_{t}\right]=\frac{1}{2}\left[\dot{x}_{t}^{*} \dot{y}_{t}+\dot{x}_{t} \dot{y}_{t}^{*}\right]-\frac{1}{4}\left[x_{t} \ddot{y}_{t}^{*}+x_{t}^{*} \ddot{y}_{t}+y_{t} \ddot{x}_{t}^{*}\right. \\
& \left.+y_{t}^{*} \ddot{x}_{t}\right]
\end{aligned}
$$

where * denotes complex conjugation. Alternatively, we define the $\Psi_{\mathrm{H}}$ operator as

$\Psi_{\mathrm{H}}\left[x_{t}, y_{t}\right]=\dot{x}_{t} \dot{y}_{t}^{*}-\frac{1}{2}\left[x_{t} \ddot{y}_{t}^{*}+\ddot{x}_{t} y_{t}^{*}\right]$

Clearly $\Psi_{\mathrm{H}}\left[y_{t}, x_{t}\right]=\Psi_{\mathrm{H}}^{*}\left[x_{t}, y_{t}\right]$ and $\Psi_{\mathrm{B}}\left[x_{t}, y_{t}\right]=\operatorname{Re}\left\{\Psi_{\mathrm{H}}\left[x_{t}, y_{t}\right]\right\}$, where $\operatorname{Re}\{\cdot\}$ denotes the real part. Both $\Psi_{\mathrm{B}}$ and $\Psi_{\mathrm{H}}$ quantify the strength of coupling or interaction between $x_{t}$ and $y_{t}$.

We also introduce the following multivariate extension of the energy operator: letting $\mathbf{z}_{1}$ and $\mathbf{z}_{2}$ denote two 
complex valued vector functions defined on $\mathbb{R}$, we let

$\Psi_{\mathrm{H}}\left[\mathbf{z}_{1 t}, \mathbf{z}_{2 t}\right]=\dot{\mathbf{z}}_{1 t} \dot{\mathbf{z}}_{2 t}^{H}-\frac{1}{2}\left[\mathbf{z}_{1 t} \ddot{\mathbf{z}}_{2 t}^{H}+\ddot{\mathbf{z}}_{1 t} \mathbf{z}_{2 t}^{H}\right]$

where ${ }^{H}$ denotes hermitian conjugation, that is, transposition plus conjugation. In particular, when $\mathbf{z}_{1}=\mathbf{z}_{2}=\mathbf{z}$, with $\mathbf{z}=[x, y]^{T}$, we get

$\Psi_{\mathrm{H}}\left[\mathbf{z}_{t}, \mathbf{z}_{t}\right]=\left[\begin{array}{ll}\Psi_{\mathrm{H}}\left[x_{t}, x_{t}\right] & \Psi_{\mathrm{H}}\left[x_{t}, y_{t}\right] \\ \Psi_{\mathrm{H}}\left[y_{t}, x_{t}\right] & \Psi_{\mathrm{H}}\left[y_{t}, y_{t}\right]\end{array}\right]$

The cross terms in the matrix $\Psi_{\mathrm{H}}\left[\mathbf{z}_{t}, \mathbf{z}_{t}\right]$ measure the coupling between $x_{t}$ and $y_{t}$ at time $t$.

The averaged $\Psi_{\mathrm{B}}$ interaction energy has been defined in [6] for finite energy signals. It follows that we can similarly define, for finite power signals, the multivariate average interaction for $\Psi_{\mathrm{H}}$ in terms of time average as

$\mathrm{E}_{\mathrm{Hz}_{1} \mathbf{z}_{2}}(\tau)=\lim _{T \rightarrow \infty} \frac{1}{2 T} \int_{-T}^{T} \Psi_{\mathrm{H}}\left[\mathbf{z}_{1 t}, \mathbf{z}_{2, t-\tau}\right] d t$.

Function $\mathrm{E}_{\mathrm{Hz}_{1} \mathbf{z}_{2}}(\tau)$ measures how similar $\mathbf{z}_{1}$ and $\mathbf{z}_{2}$ are at lag $\tau$.

As before, letting $\mathbf{z}_{1}=\mathbf{z}_{2}=\mathbf{z}$, with $\mathbf{z}=[x, y]^{T}$, we get the matrix expression of $E_{\mathrm{H}} \mathbf{z z}(\tau)$ in the form

$\mathrm{E}_{\mathrm{Hzz}}(\tau)=\left[\begin{array}{ll}\mathrm{E}_{\mathrm{H} x x}(\tau) & \mathrm{E}_{\mathrm{Hxy}}(\tau) \\ \mathrm{E}_{\mathrm{Hyx}}(\tau) & \mathrm{E}_{\mathrm{Hyy}}(\tau)\end{array}\right]$.

In particular, the cross-interaction between $x$ and $y$ is defined by

$\mathrm{E}_{\mathrm{H} x y}(\tau)=\lim _{T \rightarrow \infty} \frac{1}{2 T} \int_{-T}^{T} \Psi_{\mathrm{H}}\left[x_{t}, y_{t-\tau}\right] d t$

$\mathrm{E}_{\mathrm{H} x y}(\tau)$ quantifies the average coupling between $x_{t}$ and the delayed signal $y_{t-\tau}$.

In the following, we are going to highlight the relationship between $E_{H}$ and the correlation function. The crosscorrelation between multivariate signals $\mathbf{z}_{1}$ and $\mathbf{z}_{2}$ is defined by

$R_{\mathbf{z}_{1} \mathbf{z}_{2}}(\tau)=\lim _{T \rightarrow \infty} \frac{1}{2 T} \int_{-T}^{T} \mathbf{z}_{1 t} \mathbf{z}_{2, t-\tau}^{H} d t$.

Thus, for $\mathbf{z}_{1}=\mathbf{z}_{2}=\mathbf{z}=[x, y]^{T}$ we have

$R_{\mathbf{z z}}(\tau)=\left[\begin{array}{ll}R_{x x}(\tau) & R_{x y}(\tau) \\ R_{y x}(\tau) & R_{y y}(\tau)\end{array}\right]$

Note that $R_{y x}(\tau)=R_{x y}^{*}(-\tau)$.

Now, let us recall the following straightforward property: for two positive integers $l$ and $m$, we have

$\frac{\partial^{l+m}}{\partial \tau^{l+m}} R_{\mathbf{z}_{1} \mathbf{z}_{2}}(\tau)=(-1)^{(m)} R_{\mathbf{z}_{1}^{(l)} \mathbf{z}_{2}^{(m)}}(\tau)$

where $\mathbf{z}^{(m)}$ is the $m$ th derivative of $\mathbf{z}$. For conciseness $\mathbf{z}^{(1)}$ and $\mathbf{z}^{(2)}$ are simply denoted by $\dot{\mathbf{z}}$ and $\ddot{\mathbf{z}}$ as above. Then, we can state that

\section{Proposition 1.}

$\mathrm{E}_{\mathrm{Hz}_{1} \mathbf{z}_{2}}(\tau)=2 R_{\dot{\mathbf{z}}_{1} \dot{\mathbf{z}}_{2}}(\tau)$

In particular, for scalar signals $x$ and $y$, we get $\mathrm{E}_{\mathrm{H} x y}(\tau)=$ $2 R_{\dot{x} \dot{y}}(\tau)$.
Proof. Using relation (10), we get

$$
\begin{aligned}
\mathrm{E}_{\mathrm{Hz}_{1} \mathbf{z}_{2}}(\tau)= & \lim _{T \rightarrow \infty} \frac{1}{2 T} \int_{-T}^{T} \dot{\mathbf{z}}_{1 t} \dot{\mathbf{z}}_{2, t-\tau}^{H} \\
& -\frac{1}{2}\left[\ddot{\mathbf{z}}_{1 t} \mathbf{z}_{2, t-\tau}^{H}+\mathbf{z}_{1 t} \ddot{\mathbf{z}}_{2, t-\tau}^{H}\right] d t . \\
= & R_{\dot{\mathbf{z}}_{1} \dot{\mathbf{z}}_{2}}(\tau)-\frac{1}{2}\left[R_{\ddot{\mathbf{z}}_{1} \mathbf{z}_{2}}(\tau)+R_{\mathbf{z}_{1} \ddot{\mathbf{z}}_{2}}(\tau)\right] \\
= & 2 R_{\dot{\mathbf{z}}_{1} \dot{\mathbf{z}}_{2}}(\tau)
\end{aligned}
$$

The cross-spectrum density of $\mathbf{z}_{1}$ and $\mathbf{z}_{2}$ will be denoted by $S_{\mathbf{z}_{1} \mathbf{z}_{2}}$. It is defined as the Fourier transform of $R_{\mathbf{z}_{1} \mathbf{z}_{2}}(\tau)$ : $S_{\mathbf{z}_{1} \mathbf{z}_{2}}(f)=\mathcal{F}\left[R_{\mathbf{z}_{1} \mathbf{z}_{2}}(\tau)\right]$. In particular, for $\mathbf{z}_{1}=\mathbf{z}_{2}=\mathbf{z}=[x, y]^{T}$, we get

$S_{\mathbf{z z}}(f)=\left[\begin{array}{cc}S_{x x}(f) & S_{x y}(f) \\ S_{y x}(f) & S_{y y}(f)\end{array}\right]$

where it is clear that $S_{y x}(f)=S_{x y}^{*}(f)$, since $R_{y x}(\tau)=R_{x y}^{*}(-\tau)$. Then, we get the following result:

\section{Proposition 2.}

$\mathcal{F}\left[\mathrm{E}_{\mathrm{Hz}_{1} \mathbf{z}_{2}}(\tau)\right]=2 S_{\dot{\mathbf{z}}_{1} \dot{\mathbf{z}}_{2}}(f)$

In particular, for scalar signals $x$ and $y$, we get $\mathcal{F}\left[\mathrm{E}_{\mathrm{H} x y}(\tau)\right]=$ $2 S_{\dot{x} \dot{y}}(f)$.

Proof. From Proposition 1, $E_{\mathrm{Hz}_{1} \mathbf{z}_{2}}(\tau)=2 R_{\dot{\mathbf{z}}_{1} \dot{\mathbf{z}}_{2}}(\tau)$. Then, $\mathcal{F}\left[E_{\mathrm{Hz}_{1} \mathbf{z}_{2}}(\tau)\right]=2 \mathcal{F}\left[R_{\dot{\mathbf{z}}_{1} \dot{\mathbf{z}}_{2}}(\tau)\right]=2 S_{\dot{\mathbf{z}}_{1} \dot{\mathbf{z}}_{2}}(f)$.

It is well known that the derivation operator amounts to a filtering operation by a filter with frequency response $\hat{h}(f)=2 i \pi f$. Then, for a scalar signal $x$, we get $\mathcal{F}[\dot{x}]=\hat{h}(f)$ $\mathcal{F}[x]$. More generally, if $\mathbf{z}_{1}$ and $\mathbf{z}_{2}$ are $\mathbb{C}^{n}$ valued complex signals we obtain

$\mathcal{F}\left[\begin{array}{l}\dot{\mathbf{z}}_{1} \\ \dot{\mathbf{z}}_{2}\end{array}\right]=\hat{h}(f) \times \mathcal{F}\left[\begin{array}{l}\mathbf{z}_{1} \\ \mathbf{z}_{2}\end{array}\right]$

Then, the average interaction $E_{\mathrm{Hz}_{1} \mathbf{z}_{2}}$ can be expressed from the cross spectrum $S_{\mathbf{z}_{1} \mathbf{z}_{2}}$ as follows:

\section{Proposition 3.}

$\mathrm{E}_{\mathrm{H} \mathbf{z}_{1} \mathbf{z}_{2}}(\tau)=8 \pi^{2} \int_{\mathbb{R}} f^{2} S_{\mathbf{z}_{1} \mathbf{z}_{2}}(f) e^{2 j \pi f \tau} d f$

In particular, for scalar signals $x$ and $y$, we get the expression of $\mathrm{E}_{\mathrm{H} x y}(\tau)$ in terms of the cross-spectrum of $x$ and $y$ :

$\mathrm{E}_{\mathrm{H} x y}(\tau)=8 \pi^{2} \int_{\mathbb{R}} f^{2} S_{x y}(f) e^{2 j \pi f \tau} d f$

Proof. From Eqs. (14) and (15), we get

$$
\begin{aligned}
S_{\dot{\mathbf{z}}_{1} \dot{\mathbf{z}}_{2}}(f) & =|\hat{h}(f)|^{2} S_{\mathbf{z}_{1} \mathbf{z}_{2}}(f) \\
& =\frac{1}{2} \mathcal{F}\left[E_{\mathrm{Hz}_{1} \mathbf{z}_{2}}(\tau)\right]
\end{aligned}
$$

Then, applying the inverse Fourier transform in the above equation yields

$$
\begin{aligned}
\mathrm{E}_{\mathrm{H} \mathbf{z}_{1} \mathbf{z}_{2}}(\tau) & =2 \int_{\mathbb{R}}|\hat{h}(f)|^{2} S_{\mathbf{z}_{1} \mathbf{z}_{2}}(f) e^{2 j \pi f \tau} d f \\
& =8 \pi^{2} \int_{\mathbb{R}} f^{2} S_{\mathbf{z}_{1} \mathbf{z}_{2}}(f) e^{2 j \pi f \tau} d f
\end{aligned}
$$


Note that, up to the constant factor $8 \pi^{2}, \mathrm{E}_{\mathrm{Hz}} \mathbf{z}_{2}(\tau)$ is the second order power moment of $S_{\mathbf{z}_{1} \mathbf{z}_{2}}(f)$. Also, relation (18) yields

$S_{x y}(f)=\left(8 \pi^{2} f^{2}\right)^{-1} \mathcal{F}\left[\mathrm{E}_{\mathrm{H} x y}(\tau)\right]$

This relation suggests possible use of $\mathrm{E}_{\mathrm{H} x y}(\tau)$ for CPSD estimation purpose. In particular, the spectral coherence function between two real valued stationary zeros mean signals $x_{t}$ and $y_{t}$ is a normalized version of the cross power spectral density $S_{x y}(f)$ defined as

$\gamma_{x y}^{S}(f)=\frac{\left|S_{x y}(f)\right|}{\sqrt{S_{x x}(f)} \cdot \sqrt{S_{y y}(f)}}$.

$\gamma_{x y}^{\mathbb{S}}(f)$ takes its values in $[0,1]$. It is interesting to measure the correlation among $x$ and $y$, up to a possible filtering. Indeed, it is straightforward that $\gamma_{x y}^{\mathbb{S}}(f)$ is left unchanged through invertible filtering of $x$ or $y$ which is replaced by a filtered version of itself. Clearly, from (20) $\gamma_{x y}^{H}(f)$ can be rewritten as

$\gamma_{x y}^{H}(f)=\frac{\left|\mathcal{F}\left[\mathrm{E}_{\mathrm{H} x y}\right](f)\right|}{\sqrt{\mathcal{F}\left[\mathrm{E}_{\mathrm{H} x x}\right](f)} \cdot \sqrt{\mathcal{F}\left[\mathrm{E}_{\mathrm{H} y y}\right](f)}}$.

Note that since $\left(\gamma_{x y}^{H}(f)\right)^{2} \leq 1, \quad\left|\mathcal{F}\left[E_{\mathrm{H} x y}\right](f)\right|^{2} \leq \mathcal{F}\left[\mathrm{E}_{\mathrm{H} x x}\right](f) \cdot \mathcal{F}$ $\left[\mathrm{E}_{\text {Hyy }}\right](f)$.

When $\mathcal{F}\left[\mathrm{E}_{\mathrm{H} x y}\right](f)$ is equal to zero, $x_{t}$ and $y_{t}$ are uncorrelated at frequency $f$. At the opposite if $\mathcal{F}\left[\mathrm{E}_{\mathrm{H} x y}\right](f)$ is equal to $1, x_{t}$ and $y_{t}$ are fully correlated.

\section{Results}

In this section an application of $\Psi_{\mathrm{H}}$ for non-stationary signal analysis is presented. We show the efficiency of $\gamma_{x y}^{H}$ to estimate similarity between a signal and its noisy filtered version in the frequency domain. Let $x_{t}$ be some known (AM-FM) signal

$x_{t}=a_{t} e^{j \phi(t)}$

$a_{t}=1+\kappa \cos \left(\omega_{a} t\right)$

$\phi(t)=\left(\omega_{c} t+\omega_{m} \int_{0}^{t} q_{\tau} d \tau\right)$

$q_{t}=\sin \left(\omega_{q} t\right)$

where $a_{t}$ is the time-varying amplitude, $\omega_{c}$ the carrier frequency, $\kappa$ the AM modulation index, $q_{t}$ the frequency modulating signal and $\omega_{m}$ the maximum frequency deviation. For simulation, the parameter values used are $\omega_{q}=0.63, \omega_{a}=0.63, \omega_{c}=1.57, \omega_{m}=0.94$ and $\kappa=0.7$. Let $y_{t}$ denote the observed signal. $y_{t}$ is a noisy filtered version of $x_{t}$, where the filter impulse response is denoted by $g_{t}$ :

$y_{t}=(g * x)_{t}+n_{t}$

The additive noise $n_{t}$ is a complex circularly white Gaussian noise. For the transfer function of the filter we choose

$G(z)=1+\sum_{k=1}^{L-1} g_{k} z^{-k}$
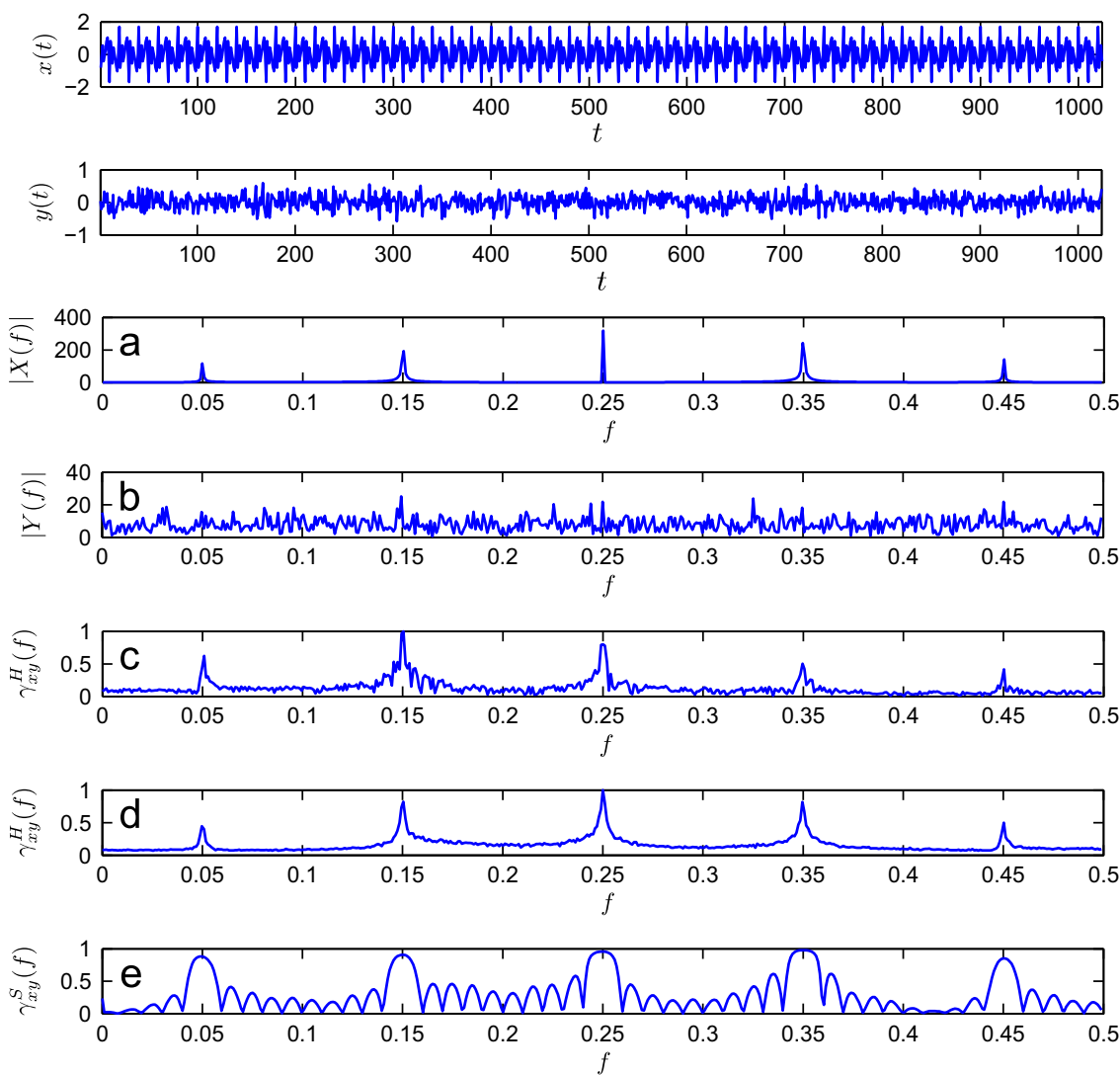

Fig. 1. Analysis of AM-FM signals. 

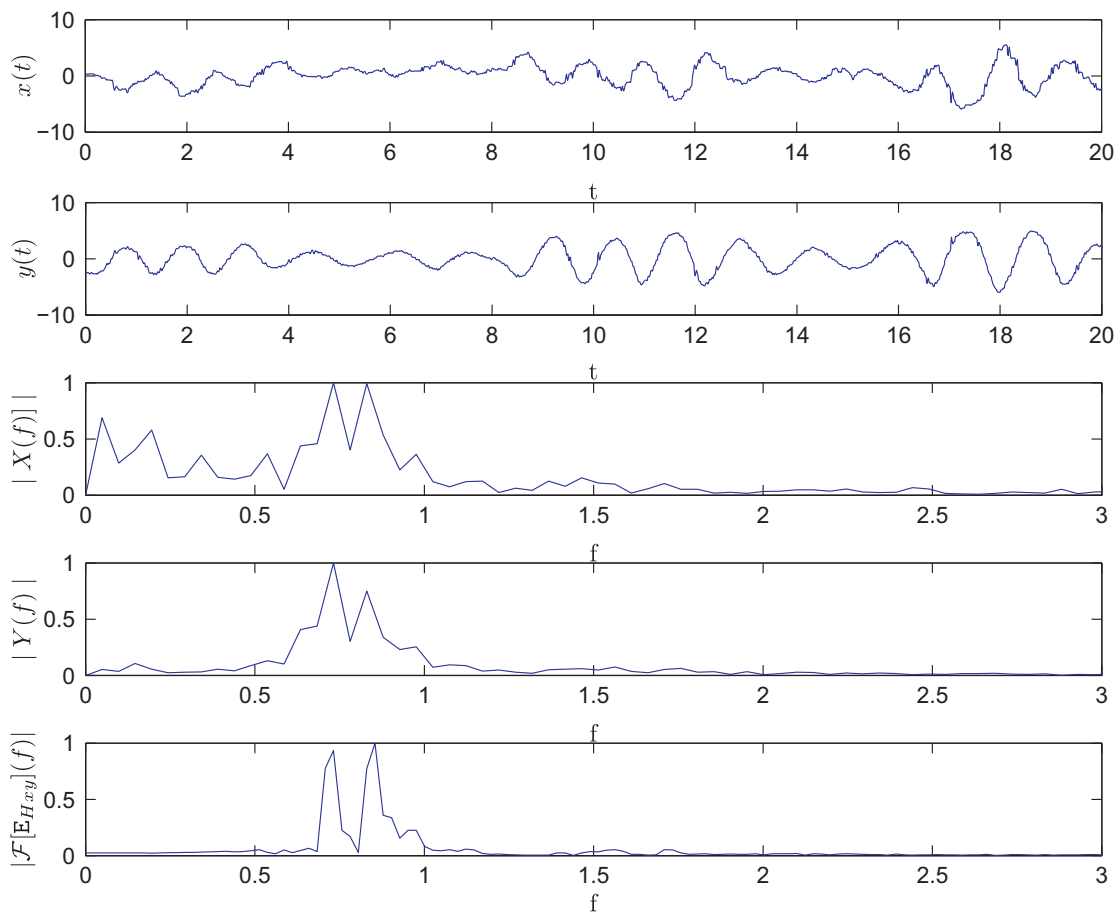

Fig. 2. Analysis of aerodynamic signals.

with $g_{k} \sim 0.1 \times \mathcal{N}(0,1)$ and $L=10$. The signal to noise ratio is set to $0 \mathrm{~dB}$ that is the mean power of $g * x$ is equal to that of the noise $n$. To detect the presence of filtered $x_{t}$ in $y_{t}$ a spectral coherence function given by Eq. (21) or Eq. (22) can be used. The spectra $\mathcal{F}\left[\mathrm{E}_{\mathrm{H} x x}(\tau)\right]$ and $\mathcal{F}\left[\mathrm{E}_{\mathrm{H} y y}(\tau)\right]$ of $x_{t}$ and $y_{t}$ and their cross spectrum $\mathcal{F}\left[E_{\mathrm{Hxy}}(\tau)\right]$ can be derived from the discrete Fourier transform. Applying Eq. (22) involves the derivation of $y_{t}$. When $n_{t}$ is a white noise, the finite difference achieves poor estimation of the derivative. There are several smoothing techniques in the literature $[10,11]$ that can be considered for derivating signals that are corrupted by white noise. The definition of $E_{H}$ in Eq. (5) involves the computation of $\Psi_{\mathrm{H}}$, and thus derivatives, but integration compensates for above mentioned difficulties and we do not need to apply sophisticated derivation techniques.

In Fig. 1(c)-(d) we plot the estimated $\gamma_{x y}^{H}(f)$ obtained after averaging over 1 and 20 realization of data of the experiment respectively. We can check that provided $\gamma_{x y}^{H}(f)$ is averaged over sufficiently many experiments, it catches well the spectral peaks of $x$ (Fig. 1(a)) in spite of the low signal to noise ratio (Fig. 1(d)). Due to its connection with spectra it is clear that the resolution of the estimated coherence increases with the length of averaged data sets while its variance decreases as the number of data sets used for its estimation increases. As for spectral estimation, the limit to distinguish frequency peaks depends on the SNR and on the amount of data available for estimation. Compared to the corresponding averaged spectra of $y$ (Fig. 1(b)) and $\gamma_{x y}^{S}(f)$ (Fig. 1(e)) we see that $\gamma_{x y}^{H}(f)$ achieves better spectral peaks detection since the effect of filter is removed in the calculation of coherence
(Fig. 1(d)). We report in Fig. 1(e) the coherence function calculated from signals spectra. This function is calculated using the MatLab function mscohere.m with the same FFT length as for the calculation from $E_{H}$ (Eq. (22)) and using rectangular window. Although spectrum-based calculation of coherence shows peaks that are often higher, the $E_{H}$ based calculation shows higher resolution.

We also illustrate the interest of $\Psi_{H}$ operator on real aerodynamic data, recorded on an instrumented yacht sailing upwind in a moderate head swell [12]. The wind signal is recorded at the top of a mast by means of a 3D acoustic anemometer that measures the instantaneous Apparent Wind Speed: $x_{t}=\operatorname{AWS}_{\dot{\theta}}(t)$. The boat pitch angle is denoted by $\theta(t)$. The corresponding pitch angle velocity is $\dot{\theta}(t) . \dot{\theta}(t)$ is recorded by a central of attitude located at the center of rotation of the boat. The pitch induces apparent wind speed $y_{t}=h \dot{\theta}(t)$ at the top of the mast, where $h$ is the height of the mast. Variations of the apparent wind speed time series $y_{t}$ are related to the frequency of waves along the boat trajectory. These variations are the reason for the aerodynamic performance oscillation of the sail plan when pitching. During a $20 \mathrm{~s}$ record (see $x_{t}$ and $y_{t}$ in Fig. 2), the swell has shown different periods. This results from the swell encountering waves at frequencies $f_{1}$ and $f_{2}$ equal to $0.73 \mathrm{~Hz}$ and $0.85 \mathrm{~Hz}$ respectively. We assume that $x_{t}$ and $y_{t}$ are ergodic processes. Frequencies $f_{1}$ and $f_{2}$ are well evidenced by $\mathcal{F}\left[\mathrm{E}_{\mathrm{H} x y}\right](f)$ (bottom plot) as common frequencies of these two signals. As it can be seen in Fig. 2, the peaks of $\mathcal{F}\left[\mathrm{E}_{\mathrm{H} x y}\right](f)$ at $f_{1}$ and $f_{2}$ show very strong correlation of $x_{t}$ and $y_{t}$ at these two frequencies. This confirms that $\Psi_{\mathrm{H}}$ is useful to show the similarities between two signals in the frequency domain. 


\section{Conclusion}

In this paper we introduced the hermitian extension of the cross $-\Psi_{\mathrm{B}}$-energy operator, denoted by $\Psi_{\mathrm{H}}$. Clearly, $\Psi_{\mathrm{H}}$ supplies a framework to study cross-energy of complexvalues signals that is more natural than $\Psi_{\mathrm{B}}$. Relationship between $\Psi_{\mathrm{H}}$ and cross-power spectral density of two complex valued signals has been established. Preliminary results on synthetic and real signals have shown the interest of $\Psi_{\mathrm{H}}$ as a similarity measure between signals. In future works, it will be interesting to investigate the use of $\Psi_{\mathrm{H}}$ for some applications where cross-energy or coherence between complex-valued signals are involved.

\section{References}

[1] J.C. Cexus, A.O. Boudraa, Link between cross-Wigner distribution and cross-Teager energy operator, Electronics Letters 40 (12) (2004) 778-780.

[2] A.O. Boudraa, J.C. Cexus, M. Groussat, P. Brunagel, An energy-based similarity measure for time series, Advances in Signal Processing (2008) 8. ID 135892.

[3] W.F. Zhang, C.C. Liu, H. Yan, Clustering of temporal gene expression data by regularized spline regression an energy based similarity measure, Pattern Recognition 43 (2010) 3969-3976.
[4] J.P. Montillet, On a novel approach to decompose finite energy functions by energy operators and its application to the general wave equation, International Mathematical Forum 48 (2010) 2387-2400.

[5] A.O. Boudraa, S. Benramdane, J.C. Cexus, Th. Chonavel, Some useful properties of cross- $\Psi_{\mathrm{B}}$-energy operator, International Journal of Electronics and Communications 63 (9) (2009) 728-735.

[6] A.O. Boudraa, J.C. Cexus, K. Abed-Meraim, Cross- $\Psi_{\mathrm{B}}$-energy operator-based signal detection, Journal of the Acoustical Society of America 123 (6) (2008) 4283-4289.

[7] A.O. Boudraa, Relationships between $\Psi_{B}$-energy operator and some time-frequency representations, IEEE Signal Processing Letters 17 (6) (2010) 527-530

[8] J.F. Kaiser, Some useful properties of Teager's energy operators, in: Proceedings of ICASSP, vol. 3, 1993, pp. 149-152.

[9] J.S. Bendat, Nonlinear System Techniques and Applications, John Wiley and Sons, Inc., NY, 1998

[10] A.V. Oppenheim, R.W. Schafer, Discrete-Time Signal Processing, Prentice Hall, 2009.

[11] A. Ditkowski, A. Bhandari, B.W. Sheldon, Computing derivatives of noisy signals using orthogonal functions expansions, Journal of Scientific Computing 36 (3) (2008) 333-349.

[12] B. Augier, P. Bot, F. Hauville, M. Durand, Experimental validation of unsteady models for fluid structure interaction: application to yacht sails and rigs, Journal of Wind Engineering and Industrial Aerodynamics 101 (2012) 53-66. 